\title{
Prognostic and Predictive Role of Angiogenic Markers in Non- Small Cell Lung Cancer
}

\author{
Serkan Keskin ${ }^{1 *}$, Ali Cevat Kutluk ${ }^{2}$, Faruk Tas ${ }^{3}$
}

\begin{abstract}
Objective: Despite the existence of detailed consensus guidelines, challenges remain regarding the role angiogenetic factors on non-small cell lung cancer (NSCLC). This study was conducted to determine the role of the vascular endothelial growth factor (VEGF), interleukin-8 (IL-8) and angiopoietin2 (Ang2) in patients with NSCLC. Methods: This study included 64 consecutive patients with non-small cell lung cancer, who admitted to clinic. Pre-treatment serum VEGF, IL-8 and Ang2 levels were evaluated. Patients were treated according to internationally accepted guidelines. Results: VEGF and IL-8 serum levels of patients with both squamous cell carcinoma and adenocarcinoma were significantly higher than controls $(\mathrm{p}<0.05)$. In addition, IL-8 levels were lower among treatment-responders than non-responders ( $\mathrm{p}: 0.031)$. Impact of elevated or decreased levels of VEGF, Ang2 and IL-8 on survival was evaluated, accepting median level as reference. There was no correlation between the serum levels of VEGF, Ang2, IL-8 and survival. Conclusion: We found that the levels of angiogenic markers were significantly different between non-small cell lung cancer patients and controls. These markers could elicit more information related to stage and prognosis.
\end{abstract}

Keywords: Non-small cell lung cancer- angiogenesis- stage- prognosis

Asian Pac J Cancer Prev, 20 (3), 733-736

\section{Introduction}

Lung cancer ranks among the most commonly occurring malignancies and currently is the leading cause of cancer related deaths in worldwide. Non-small cell lung cancer is the histologic diagnosis in approximately 80 percent of the patients diagnosed with lung cancer.

The malignant phenotype of lung cancer may be partially attributable to abnormalities in growth factors and their receptors acting via both autocrine and paracrine pathways. An understanding of the abnormal expression of such growth factors is crucial to find new therapeutic targets (Tas et al., 2006).

Tumor vascularization is crucial for the pathogenesis of all solid malignancies. In the absence of local capillary proliferation, tumors may not grow beyond 2-3 $\mathrm{mm}$ in diameter (Smith et al., 1994). Certain tumors have been found to produce factors that are directly angiogenic, whereas others may depend upon vascularization induced by products of responding inflammatory cells.

The angiogenic phenotype depends on a net balance between positive and negative angiogenic factors released by the tumor. So far, many angiogenic factors, such as vascular endothelial growth factor (VEGF), interleukin-8 (IL-8), and angiopoietin2 (Ang2) have been identified and shown to be produced by a variety of different tumor cells. However, a limited number of studies investigated correlation between stages of lung cancer, response to treatment and levels of angiogenic markers.

In this study, we compared the serum angiogenic factors including VEGF, Ang2 and IL-8 in patients with NSCLC and healthy controls. Moreover, we investigated levels of angioenic markers and response to treatment.

\section{Materials and Methods}

We studied 64 patients consecutively recently histological or cytologically verified diagnosed of NSCLC. All of the patients were treated according to internationally accepted guidelines. Response to chemotherapy was determined after 2 or 3 cycles of chemotherapy according to standard RECIST criteria. The treatment was interrupted in progressed patients after response evaluation.

Normal controls $(n=29)$ were recruited from among the Institute personnel and were selected based on identical to patients such as, age, gender, smoking status, and presence of various non-malignant pulmonary disorders. All patients gave informed consent and the protocol was approved by the Institutional Review Board.

\section{Measurement of serum VEGF, Ang2 and IL-8 levels}

Blood samples were obtained within one weeks before chemotherapy in fasting conditions and serum was frozen at $-20^{\circ} \mathrm{C}$ for further determination. 
VEGF (InvitrogenR), İL-8 (InvitrogenR) and Ang2 (R and D Systems Inc. MN55413, U.S.A.) kit is a solid phase sandwich Enzyme Linked-Immuno-Sorbent Assay (ELISA). A monoclonal antibody specific for markers has been coated onto the wells of the microtiter strips provided. Samples, control specimens and unknowns are pipetted into these wells. During the first incubation, the antigen and a biotinylated polyclonal antibody specific for markers are simultaneously incubated. After washing, the streptavidin- peroxydase is added. After incubation and washing to remove all the unbound enzyme, a substrate solution, which is acting on the bound enzyme, is added to induce a colored reaction product.

\section{Study evaluations}

Pretreatment evaluations was including a complete medical history and physical examination, hematologic and biochemical profiles, electrocardiogram, chest X-ray, whole bone scan, computed tomography scan of the chest and upper abdomen, and magnetic resonance imaging of the whole brain.

\section{Eligibility Criteria}

Patients who had received prior chemotherapy were ineligible. Likewise, patients with poor performance status (PS) of 3 or 4 were also not enrolled into the study. However, the presence of brain metastasis and older age were not defined as exclusion criteria, therefore, these patients were accepted for the trial. Patients had adequate bone marrow, hepatic, renal functions (defined, respectively, as a WBC count $\geq 3.500$ cells $/ \mu \mathrm{L}$, an absolute granulocyte count $\geq 2.000$ cells $/ \mu \mathrm{L}$, and a platelet count $\geq 100.000$ cells $/ \mu \mathrm{L}$; a serum bilirubin level $\leq 1.5 \mathrm{mg} / \mathrm{dL}$; and a serum creatinine level $\leq 1.5 \mathrm{mg} / \mathrm{dL}$ or creatine clearance $\geq 60 \mathrm{ml} / \mathrm{min}$ ), and no active infections, cardiac or metabolic disease.

\section{Response and Toxicity Evaluation}

Tumor response was classified by standard RECIST criteria. A complete response (CR) was defined as the complete disappearance of all clinically detectable tumors for at least 4 weeks. A partial response (PR) was defined as an at least $30 \%$ decrease in the sum of the longest diameters of the target lesions for more than 4 weeks with no new area of malignant disease. Progressive disease (PD) indicated at least a $20 \%$ increase in the sum of the longest diameter of target lesions or a new malignant lesion. Stable disease (SD) was defined as insufficient shrinkage to qualify for PR and insufficient increase to qualify for PD. Toxicity evaluations were performed based on the National Cancer Institute's Common Terminology Criteria for Adverse Events version 4.0.

\section{Statistical Analysis}

All analysis were performed by using the SPSS 15.0 statistical software package (Chicago, IL), with $\mathrm{P}<0.05$ considered to be significant. Patient characteristics were tabulated or described by their median and range. Mann-Whitney U test was used to compare groups. Primary endpoint was defined as progression free survival and secondary end point was defined as overall survival and overall response rate. Progression free survival in former studies with same triplet regimen showed a success of 6 months. We included new patients in our study until we reached a $95 \%$ confidence interval rate including minimum 6 months. We stopped new patient's admission after the first patient had a one year follow-up. Sample size was determined using Simon's tables.

Overall survival (OS) was measured from the histologic diagnosis until death or last day of clinical visit. Progression-free survival (PFS) was measured from the histological diagnosis until the first objective evidence of tumor progression. Survival distributions were estimated with the Kaplan-Meier method. Cox's proportional hazard regression models were used to assess the prognostic significance of clinical and histopathological characteristics of the tumor on outcomes. Factors that were statistically significant at the univariate analysis were entered into the multiple regression models. Results from Cox's models were expressed as hazard ratios (HRs) with 95\% confidence intervals (CIs). Surivival times were represented by mean instead of median due to because of the number of events some groups is less than calculated levels.

\section{Results}

This study includes 64 patients and 29 healty persons. Age and gender are not different between patietns and control groups. Median age for patietns NSCLC was $60 \pm 9$

Table 1. Marker Levels on Patients and Controls, SI

\begin{tabular}{lcccccc}
\hline & VEGF & $\mathrm{p}$ & $\mathrm{Ang} 2$ & $\mathrm{p}$ & $\mathrm{IL}-8$ & $\mathrm{p}$ \\
\hline NSCLC & $144 \pm 127(1.7-518)$ & $<0.001$ & $1.755 \pm 2.3(0.77-15)$ & 0.212 & $13 \pm 43(0.5-301)$ & 0.035 \\
Control & $36 \pm 40(19-177)$ & & $1.7 \pm 0.7(0.8-3.6)$ & & $8 \pm 22(0.2-81)$ & \\
Squamous & $125 \pm 129(1.7-518)$ & $<0.001$ & $2.09 \pm 2.7(1-15)$ & 0.032 & $14 \pm 29(3.6-141)$ & 0.064 \\
Adenocarcinoma & $161 \pm 125(6.6-503)$ & & $1.5 \pm 1.7(0.77-8)$ & & $11 \pm 54(0.5-301)$ & \\
Control & $36 \pm 40(19-177)$ & & $1.7 \pm 0.7(0.8-3.6)$ & & $8 \pm 22(0.2-81)$ & \\
Squamous & $125 \pm 129(1.7-518)$ & 0.184 & $2.09 \pm 2.7(1-15)$ & 0.024 & $14 \pm 29(3.6-141)$ & 0.28 \\
Adenocarcinoma & $161 \pm 125(6.6-503)$ & & $1.5 \pm 1.7(0.77-8)$ & & $11 \pm 54(0.5-301)$ & \\
Squamous & $125 \pm 129(1.7-518)$ & $<0.001$ & $2.09 \pm 2.7(1-15)$ & 0.023 & $14 \pm 29(3.6-141)$ & 0.023 \\
Control & $36 \pm 40(19-177)$ & & $1.7 \pm 0.7(0.8-3.6)$ & & $8 \pm 22(0.2-81)$ & \\
Adenocarcinoma & $161 \pm 125(6.6-503)$ & $<0.001$ & $1.5 \pm 1.7(0.77-8)$ & 0.925 & $11 \pm 54(0.5-301)$ & 0.161 \\
Control & $36 \pm 40(19-177)$ & & $1.7 \pm 0.7(0.8-3.6)$ & & $8 \pm 22(0.2-81)$ & \\
\hline
\end{tabular}


Table 2. Marker Levels and Response to Therapy, SI

\begin{tabular}{lccc}
\hline & Responder $(\mathrm{CR}+\mathrm{PR})$ & Non-Responder (SD + PD) & $\mathrm{p}$ \\
$\mathrm{n}=34$ & $183 \pm 128(47-518)$ & 0.11 \\
VEGF & $119 \pm 124(1.7-491)$ & $2.2 \pm 3(1.06-15)$ & 0.1 \\
Ang2 & $1.57 \pm 1.36(0.77-6.5)$ & $34 \pm 33(0.5-141)$ & 0.031 \\
IL-8 & $10.9 \pm 51(2.8-301)$ & . & \\
\hline
\end{tabular}

Abbreviations, CR, complete response; PR, partial response; SD, stabile disease; PD, progressive disease.

Table 3. The Role of Angiogenic Markers on Overall Survival, Months

\begin{tabular}{|c|c|c|c|c|c|c|c|}
\hline & Level, SI & $\mathrm{n}$ & Event & Mean & $\mathrm{SD}$ & $\% 95 \mathrm{CI}$ & $\mathrm{p}$ \\
\hline \multirow[t]{3}{*}{ VEGF } & & & & & & & 0.809 \\
\hline & $<100$ & 16 & 2 & 26.2 & 3.1 & $20.2-32.2$ & \\
\hline & $\geq 100$ & 41 & 6 & 24.3 & 2.1 & $20.2-28.3$ & \\
\hline \multirow[t]{3}{*}{ Ang2 } & & & & & & & 0.142 \\
\hline & $<1.5$ & 22 & 1 & 27.8 & 2.1 & $23.7-31.8$ & \\
\hline & $\geq 1.5$ & 35 & 7 & 23.3 & 2.5 & $18.4-28.1$ & \\
\hline \multirow[t]{3}{*}{ IL-8 } & & & & & & & 0.092 \\
\hline & $<10$ & 17 & 1 & 28.3 & 1.6 & $25.1-31.4$ & \\
\hline & $\geq 10$ & 40 & 7 & 21.9 & 2.9 & $16.3-27.5$ & \\
\hline
\end{tabular}

(35-77) years, the patients had adenocarcimona histology was $58 \pm 8$ (42-74) years, and the patients had squamous cell carcinoma histology was $63 \pm 9$ (35-77) years. Eight patients $(12.5 \%)$ were female, 28 patients $(44 \%)$ has stage III diesease, 36 patients ( $56 \%$ ) has stage IV disease, 32 patients $(50 \%)$ has adenocarcinoma histology, 30 patients $(83 \%)$ has one metastatic site.

The median serum levels of VEGF $(p<0.001)$ and IL- 8 (p: 0.035) were significantly increased in NSCLC patients compared with healthy controls (Table 1). VEGF and IL-8 levels is higher adenocarcinoma patitents compared to squamous cell carcinoma. Inversely, Ang-2 levels is higher on patients with squamous cell carcinoma.

IL-8 levels is higher for responder patients compared to non-responder (p:0.031). VEGF and Ang-2 levels are not different between responder and non-responder patients (Table 2).

The median survival of all patient groups was 223 days $(21-967 \pm 250)$. With the median serum angiogenic levels as the cut-off value, patients were divided into high and low serum parameter groups. There is no correlation between VEGF, Ang-2 and IL-8 levels and survival for all patients (Table 3 and Table 4). Similarly, angiogenic markers are not play a role on survival for adenocarcinoma and squamous cell carcinoma patients.

\section{Discussion}

In the present study, we evaluated the serum concentration and the prognostic significance of three of important angiogenic factors in NSCLC patients. We found significantly elevated serum levels of VEGF and IL-8 in NSCLC patients compared with healthy controls. Also, marker levels are important for adenocarcinoma and squamous histology. IL-8 levels is more important for squamous histology compared to adenocarcinoma. Furthermore, elevated serum levels of angiogenic factors were not found correlated with poor overall survival except IL-8 levels for squamous histology.

We and others have previously shown elevated concentrations of several circulating cytokines and soluble cytokine receptors in patients with NSCLC (Kaminska et al., 2006; Tas et al., 2006; Waugh and Wilson, 2008; Caporarello et al., 2017). A growing body of data confirms the role of the inflammatory component with its mediators in cancer development and progression. Our present

Table 4. The Role of Angiogenic Markers on Progression Free Survival, Months

\begin{tabular}{|c|c|c|c|c|c|c|c|}
\hline & Levels. SI & $\mathrm{n}$ & Event & Mean & Std dev & $\% 95 \mathrm{CI}$ & $\mathrm{p}$ \\
\hline \multirow[t]{3}{*}{ VEGF } & & & & & & & 0.231 \\
\hline & $<100$ & 17 & 6 & 19 & 3.6 & $12.1-25.9$ & \\
\hline & $\geq 100$ & 41 & 22 & 14.5 & 2.1 & $10.5-18.5$ & \\
\hline \multirow[t]{3}{*}{ Ang2 } & & & & & & & 0.257 \\
\hline & $<1.5$ & 22 & 13 & 12.6 & 2.7 & $7.3-17.9$ & \\
\hline & $\geq 1.5$ & 36 & 15 & 17.8 & 2.4 & $13.1-22.5$ & \\
\hline \multirow[t]{3}{*}{ İL-8 } & & & & & & & 0.291 \\
\hline & $<10$ & 17 & 8 & 18.5 & 3.1 & $12.6-24.4$ & \\
\hline & $\geq 10$ & 41 & 20 & 14.5 & 2.3 & $10.1-19.1$ & \\
\hline
\end{tabular}


and previous studies on circulating cytokines in cancer patients are in accordance with this observation, since the cytokines that were most commonly linked to the clinicopathological features of tumors were those often associated with inflammatory reactions.

VEGF, one of the most potent angiogenic factor, has an important role in the process of human angiogenesis (Poon et al., 2001). The finding of significantly elevated serum VEGF levels in cancer patients than normal humans was reported in many studies (Yamamoto et al., 1996). Serum levels of VEGF have been correlated with the extent of disease or poor response to chemotherapy (Viallard and Larrivee, 2017). High serum and tumor levels of VEGF have been associated with poorer survival in some, but not all, studies.

Interleukin-8/CXCL8 (IL-8) is a member of the CXC chemokine family. The angiogenic activity of IL-8 produced by monocytes and macrophages was first demonstrated in 1992 (Koch et al., 1992). IL-8 is a potent angiogenic factor in several carcinomas including lung cancer. The researchers found that increased quantities of IL-8 were detected in tumor tissue as compared with normal lung tissue (Yuan et al., 2000; Masuya et al., 2001). One of the most impoartant results for our study, histology play a more important role for angiogenic markers. In the future, for this results may be critical role for immunotherapy and squamous cell carcinoma therapy (Alfaro et al., 2017). Given the fact that in cancer patients IL- 8 is mainly produced by tumor cells themselves, its serum concentration has been shown to correlate with tumor burden. Thus, IL-8 serum concentrations have been shown to be useful as a pharmacodynamic biomarker to early detect response to immunotherapy. Finally, because of the roles that IL-8 plays in favoring tumor progression, several therapeutic strategies are being developed to interfere with its functions (Khan et al., 2015; Alfaro et al., 2017).

In conclusion, we found that the levels of circulating angiogenic markers were significantly higher in NSCLC patients compared with healthy controls. Additionally, angiogenic markers are different between different histology. Also, angionenic markers may be play a rola for survival for these patients. Additional investigations contain larger SCLC patients are needed to confirm these findings. Understanding the biologic role of circulating angiogenic factors in cancer patients may provide insight into potential strategies to prevent the development of metastasis. This opens up a potential role of antiangiogenic therapy and immunotherpy in suppressing the development of metastasis.

\section{References}

Alfaro C, Sanmamed MF, Rodriguez-Ruiz ME, et al (2017). Interleukin-8 in cancer pathogenesis, treatment and follow-up. Cancer Treat Rev, 60, 24-31.

Caporarello N, Lupo G, Olivieri M, et al (2017). Classical VEGF, Notch and Ang signalling in cancer angiogenesis, alternative approaches and future directions (Review). Mol Med Rep, 16, 4393-402.

Kaminska J, Kowalska M, Kotowicz B, et al (2006). Pretreatment serum levels of cytokines and cytokine receptors in patients with non-small cell lung cancer, and correlations with clinicopathological features and prognosis. M-CSF - an independent prognostic factor. Oncology, 70, 115-25.

Khan MN, Wang B, Wei J, et al (2015). CXCR1/2 antagonism with CXCL8/Interleukin-8 analogue CXCL8(3-72)K11R/ G31P restricts lung cancer growth by inhibiting tumor cell proliferation and suppressing angiogenesis. Oncotarget, 6, 21315-27.

Koch AE, Polverini PJ, Kunkel SL, et al (1992). Interleukin-8 as a macrophage-derived mediator of angiogenesis. Science, 258, 1798-801.

Masuya D, Huang C, Liu D, et al (2001). The intratumoral expression of vascular endothelial growth factor and interleukin- 8 associated with angiogenesis in nonsmall cell lung carcinoma patients. Cancer, 92, 2628-38.

Poon RT, Fan ST, Wong J (2001). Clinical implications of circulating angiogenic factors in cancer patients. J Clin Oncol, 19, 1207-25.

Smith DR, Polverini PJ, Kunkel SL, et al (1994). Inhibition of interleukin 8 attenuates angiogenesis in bronchogenic carcinoma. J Exp Med, 179, 1409-15.

Tas F, Duranyildiz D, Oguz H, et al (2006). Serum vascular endothelial growth factor (VEGF) and bcl-2 levels in advanced stage non-small cell lung cancer. Cancer Invest, 24, 576-80.

Viallard C, Larrivee B (2017). Tumor angiogenesis and vascular normalization: alternative therapeutic targets. Angiogenesis, 20, 409-26.

Waugh DJ, Wilson C (2008). The interleukin-8 pathway in cancer. Clin Cancer Res, 14, 6735-41.

Yamamoto Y, Toi M, Kondo S, et al (1996). Concentrations of vascular endothelial growth factor in the sera of normal controls and cancer patients. Clin Cancer Res, 2, 821-6.

Yuan A, Yang PC, Yu CJ, et al (2000). Interleukin-8 messenger ribonucleic acid expression correlates with tumor progression, tumor angiogenesis, patient survival, and timing of relapse in non-small-cell lung cancer. Am J Respir Crit Care Med, 162, 1957-63.

This work is licensed under a Creative Commons AttributionNon Commercial 4.0 International License. 